\title{
Calidad de los recursos hídricos en el contexto de la actividad económica y patrones de salud en Sonora, México
}

\author{
Water resource quality as related to economic activity \\ and health patterns in Sonora, Mexico
}

${ }^{1}$ Doctor en Economía. Profesor-Investigador, El Colegio de la Frontera Norte, Tijuana, México. $\Delta$ (iD)
RESUMEN El objetivo del presente trabajo es analizar la distribución espacial de fuentes potenciales de contaminación de los recursos hídricos en el contexto de la actividad económica, y proponer una regionalización en relación con la ocurrencia de defunciones por cáncer en el estado fronterizo de Sonora, México. La metodología se basa en análisis exploratorio e inferencial de datos, en el que se utilizan dos fuentes de datos primarias: los aprovechamientos por descargas de agua residuales inscritos en el Registro Público de Derechos de Agua (REPDA) y los registros generados por el Sistema Nacional de Información en Salud (SINAIS) en el periodo 1998-2011 basado en la Clasificación Internacional de Enfermedades (CIE-10). El análisis de concentración espacial propuesto permite identificar causas específicas de defunción por cáncer a nivel regional. Los resultados indican que es de interés para la región el ajuste de los parámetros de calidad proyectados por la Norma Oficial Mexicana NOM-250-SSA1-2014 que consigna un subconjunto de contaminantes comunes en sitios con actividad minera.

PALABRAS CLAVES Neoplasias; Recursos Hídricos; México.

\begin{abstract}
The aim of this work is to analyze the spatial distribution of potential pollution pathways of water resources given the economic activity in the Mexican border state of Sonora and propose a regional distribution in relation to cancer mortality rates across the state. The methodology is based in an exploratory and inferential data analysis using two sources of primary data: wastewater discharge concessions registered in the Public Registry on Water Rights [Registro Público de Derechos de Agua] (REPDA) and the records generated by the National Health Information System [Sistema Nacional de Información en Salud] (SINAIS) in the period 1998-2011 based on the International Classification of Disease (ICD-10). The spatial concentration analysis allows for the identification of specific cancer mortality causes at the regional level. Results indicate that the projected adjustments to the regulation NOM-250-SSA1-2014, which controls a subset of pollutants common in mining activity surroundings, is a matter of regional concern.
\end{abstract}

KEY WORDS Neoplasms; Water Resources, Mexico. 


\section{INTRODUCCIÓN}

El estado mexicano de Sonora, ubicado en la frontera con EE.UU. ha registrado históricamente una intensa actividad minera ${ }^{(1,2,3,4,5)}$, una vocación productiva que se extiende a través de la frontera ${ }^{(6)}$ y que ha definido la conformación de las relaciones humanas en la región binacional entre Arizona y Sonora ${ }^{(7,8,9,10,11)}$.

En la relación medio ambiente y economía, la relevancia de la minería en la región fronteriza de Sonora y Arizona se debe a que allí se encuentra la principal zona de extracción de cobre en México. Esto ha permitido el establecimiento de 58 sitios productores de este mineral y los dos complejos mineros más grandes del país: Buenavista del Cobre y La Caridad, ubicados en los municipios de Cananea y Nacozari respectivamente.

En el contexto de la bibliografía que aborda la relación entre externalidades ${ }^{[a]}$ derivadas de la actividad económica y los efectos en salud, un factor relevante por su correlación es la exposición prolongada a contaminantes comunes en sitios con alta presencia de actividad minera. Esta línea de investigación reconoce que entender los determinantes sociales de la salud es una prioridad global ${ }^{(12)}$.

En actividades económicas como la minería, y en la extracción de cobre en particular, el proceso conocido como drenado ácido y otras descargas representa un foco de atención. Este proceso implica la filtración de residuos y la absorción en el sedimento de metales pesados como cadmio $(\mathrm{Cd})$, cromo $(\mathrm{Cr})$, plomo $(\mathrm{Pb})$, hierro $(\mathrm{Fe})$ y metaloides, como arsénico (As), entre otros elementos ${ }^{(13,14)}$.

De estos elementos químicos, el arsénico es un componente de interés ${ }^{(15,16)}$ debido a que puede ser liberado por actividades antropogénicas, como la apertura de tajos implícita en la minería del cobre, aunque es un elemento de ocurrencia natural común en regiones áridas y semiáridas como la región fronteriza entre Sonora y Arizona ${ }^{(17)}$. Desde la década de 1980, diversos estudios demuestran que la exposición crónica a dosis elevadas de arsénico, en su forma inorgánica, se asocia con el desarrollo de cáncer y otros efectos adversos a la salud, incluidos padecimientos cutáneos ${ }^{(18,19,20)}$; siendo el agua para beber una de las vías principales de exposición humana a este elemento(21).

De forma paralela, durante la última década, en este estado fronterizo, la incidencia de padecimientos como cáncer ha registrado un incremento del $44 \%$ al pasar de 1.594 en 1998 a 2.299 casos en 2011, con una tasa de crecimiento promedio anual del $3 \%$, superior al ritmo de crecimiento anual de la tasa de mortalidad que fue del $1,1 \%$.

Si bien se reconoce que el mecanismo de contaminación de las fuentes de agua es complejo e intervienen múltiples factores ${ }^{(22)}$, la evidencia de estudios en este sentido muestra que ciertas actividades de origen antropogénico, como la minería, tienen el potencial de incrementar el riesgo de exposición de las fuentes de abastecimiento por vía de contaminación del subsuelo o al contaminar las fuentes someras de abastecimiento del agua de los centros poblacionales, como los ríos ${ }^{(23)}$.

Más aún, se reconoce que la exposición a residuos químicos derivados de estos procesos productivos constituye un riesgo potencial para la salud por su persistencia en el entorno $^{(24)} y$, en el caso de los residuos depositados en las inmediaciones de sitios con actividad minera, la interacción con las corrientes de agua superficial representa un mecanismo de transmisión que posibilita la exposición de la población a estos elementos.

Dado este contexto, la calidad de los recursos hídricos es un tema que genera interés ante la ocurrencia de eventos como el derrame de $40 \mathrm{mil} \mathrm{m}^{3}$ de una solución de sulfato de cobre $\left(\mathrm{CuSO}_{4}\right)$ en las inmediaciones del río Bacanuchi, afluente del río Sonora, en agosto de $2014^{(25)}$, contingencia que derivó, en un inicio, en la sanción económica más alta de la historia impuesta por la legislación ambiental en México ${ }^{(26)}$.

Según se documentó, la solución vertida contenía la siguiente concentración de metales: hierro $1.080 \mathrm{mg} / \mathrm{l}$, aluminio $461 \mathrm{mg} / \mathrm{l}$, 
cobre $141 \mathrm{mg} / \mathrm{l}$, manganeso 98,1 mg/l, zinc $51 \mathrm{mg} / \mathrm{l}$, arsénico $42,7 \mathrm{mg} / \mathrm{l}$, niquel $11 \mathrm{mg} / \mathrm{l}$, cadmio $7,76 \mathrm{mg} / \mathrm{l}$, plomo $2,5 \mathrm{mg} / \mathrm{l}$, cromo $1,54 \mathrm{mg} / \mathrm{I}^{(27)}$; elementos químicos cuyo impacto adverso sobre la salud es reconocido ante exposiciones prolongadas y se consigna dentro de la Norma Oficial Mexicana NOM-127-SSA1-1994, en el ámbito de salud ambiental ${ }^{(28)}$. La Figura 1 muestra la localización del área estudiada en el presente trabajo.

A pesar de este nexo entre los factores de exposición ambientales desde la perspectiva de salud pública, en el norte de México, el estudio de la relación entre factores ambientales de exposición de origen antropogénico e indicadores de salud desagregados a nivel geográfico es aún limitado. Sin embargo, la existencia de microdatos geográficamente referenciados, generados por el Sistema Nacional de Información en Salud (SINAIS), representa una fuente de información valiosa para evaluar esta relación en la región fronteriza entre Sonora y Arizona.

El objetivo del presente trabajo es analizar la distribución espacial de fuentes potenciales de contaminación de los recursos hídricos, proponer una regionalización basada en las características hidrológicas del estado y realizar un análisis exploratorio e inferencial de datos en relación con la ocurrencia de defunciones por cáncer en el estado de Sonora.

Se pretende que el análisis contribuya a generar una base de referencia para estudios posteriores a la contingencia ambiental ocurrida en Sonora, México en el año 2014, que presenta un reto por la complejidad de los factores involucrados. De acuerdo con órganos regulatorios en materia de protección contra riesgos sanitarios, es recomendable un seguimiento epidemiológico de largo plazo ${ }^{(25)}$.

El marco conceptual de partida se basa en el estudio de los determinantes sociales de las condiciones de salud de la población a partir de una concepción geoespacial. Este marco de análisis permite vincular el desarrollo de actividades económicas específicas a nivel local con la distribución de eventos en salud.

El enfoque adoptado se sustenta en dos pilares teóricos: el primero es la noción de salud ambiental delineada por la Organización Mundial de la Salud como "aquellos aspectos de la salud humana incluyendo calidad de vida, que son determinados por factores químicos, físicos, biológicos, sociales y sicosociales en el medioambiente" ${ }^{\prime \prime 29}$

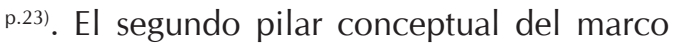
teórico propuesto es el paradigma de epidemiología social. Tal como apunta Krieger ${ }^{(30)}$, una de las críticas al canon de la epidemiología clásica contemporánea representado por el modelo de causalidad múltiple es su dependencia del individualismo médico. Este paradigma concede a los métodos epidemiológicos una posición predominante sobre las teorías de la causalidad de los padecimientos, sin embargo, un enfoque alternativo plantea complementar el paradigma clásico con una visión sobre "la producción social de los padecimientos" $^{\prime \prime 30}$ p.894) que no se concentre únicamente en la esfera biológica ${ }^{(31)}$.

La aplicación de este enfoque teórico ha tenido particular aceptación a nivel global para entender el vínculo entre salud y actividades económicas como la minería, área en

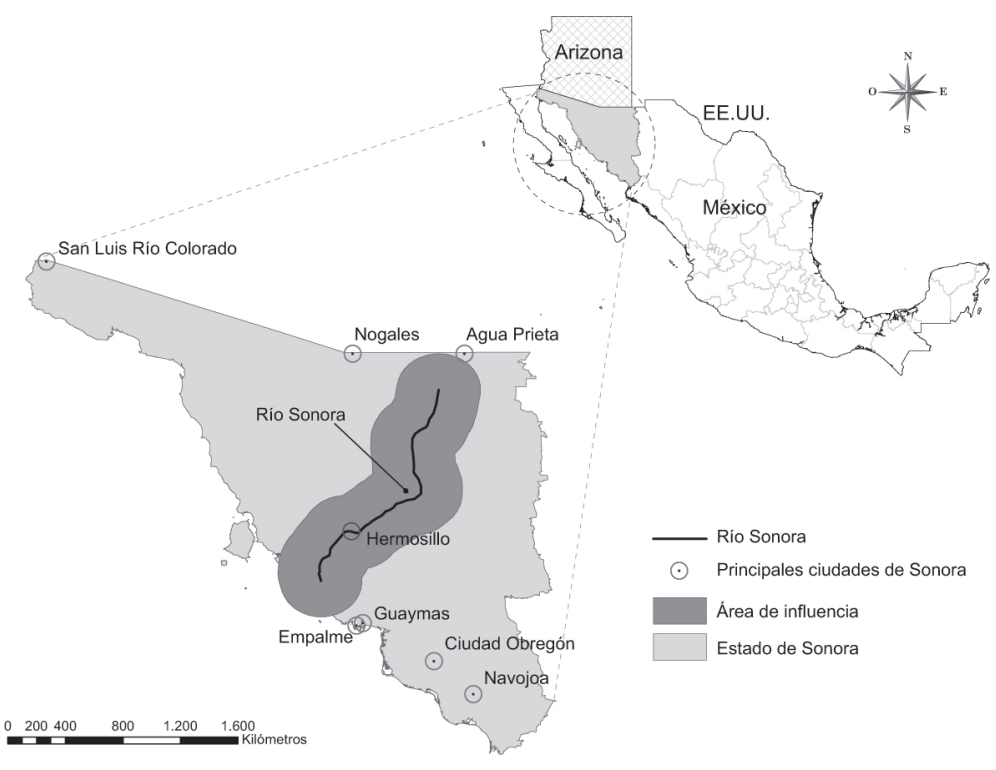

Figura 1. Localización del área de estudio. Estado de Sonora, México.

Fuente: Elaboración propia a partir de datos del Instituto Nacional de Estadística y Geografía (INEGI) y la Comisión Nacional para el Conocimiento y Uso de la Biodiversidad (CONABIO). 
la que prevalece un entendimiento rudimentario a pesar del creciente interés social por indagar esta relación. El auge de la investigación en esta área sugiere que la incorporación de los aspectos geográficos contribuye a comprender los mecanismos de exposición e incidencia ${ }^{(24,32,33)}$ así como al diseño de esquemas de evaluación de largo plazo ante la ocurrencia de contingencias. Por ello, se considera que la aplicación de este marco conceptual podría aportar elementos sobre los determinantes sociales en el contexto de la actividad económica en el estado fronterizo de Sonora en el norte de México.

\section{MÉTODOS}

El estudio se basa en un análisis exploratorio e inferencial de datos. El análisis se divide en dos fases: en la primera se aplican técnicas de análisis espacial para determinar la relación de la actividad económica y los aprovechamientos inscritos en el Registro Público de Derechos de Agua en la categoría descargas de agua residual.

Este nivel de análisis es relevante por las implicaciones sociales asociadas a la calidad de los recursos hídricos superficiales del estado. Se adopta como unidad de análisis la delimitación por cuencas hidrográficas. En esta vertiente se aplican herramientas geoestadísticas con el método probabilístico Kriging $^{(34)}$, que retoman el principio de regresión de mínimos cuadrados ordinarios en el contexto geográfico ${ }^{(35)}$ para construir una superficie que muestra la densidad espacial de las concesiones por descarga de agua residual, lo que permite identificar áreas de atención a partir de clústeres de descargas.

Para este propósito se construyó un modelo geoestadístico de probabilidad Kriging, que parte de aplicaciones como Waller y Gotway ${ }^{(36)}$ que determina la probabilidad de exceder el volumen promedio concesionado en el estado, contemplando la distribución geográfica de las concesiones a partir de la función de correlación espacial, y permite construir una superficie que muestra la concentración.
El modelo asume la siguiente forma funcional:

$\mathrm{I}(\mathrm{s})=\mathrm{I}(\mathrm{Z}(\mathrm{s})>\mathrm{ct})=\mu 1+\varepsilon 1(\mathrm{~s}), \mathrm{Z}(\mathrm{s})=\mu 2+\varepsilon 2(\mathrm{~s})$

Donde: I(s) es una variable binaria que toma el valor de 1 si se excede el volumen concesionado promedio, y 0 en caso contrario; $\mathrm{I}(\mathrm{Z}(\mathrm{s})>\mathrm{ct})$ umbral de referencia; $\mathrm{Z}(\mathrm{s})$ volumen concesionado en la localización $s ; \mu 1$ y $\mu 2$ constantes; y $\varepsilon 1, \varepsilon 2$ errores estocásticos.

La fuente de datos de este primer apartado es el Registro Público de Derechos de Agua, generado por la Comisión Nacional de Agua (CONAGUA) ${ }^{(37)}$ que permite contar con información georreferenciada de las concesiones por descargas de aguas residuales en el estado. Esta base reporta 642 concesiones con variables como el volumen concesionado y el tipo de descarga, lo que permite asociar el impacto con actividades económicas específicas a lo largo de los 72 municipios de la entidad. El objetivo del primer apartado es construir una regionalización a partir de la cual explorar indicadores de salud en una segunda etapa de la investigación.

En la segunda fase se realizó un análisis exploratorio e inferencial de datos utilizando los registros generados por el Sistema Nacional de Información en Salud (SINAIS) en el periodo 1998-2011. Esta base permite estudiar los patrones espaciales de las defunciones por cáncer en el estado de Sonora. La base de datos retoma estándares internacionales ya que está construida a partir de la Clasificación Internacional de Enfermedades (CIE-10), lo que permite generar indicadores comparables respecto al contexto internacional.

Se consideraron 14.752 registros para el año 2011 y, dentro de estos, se identificaron aquellas causas clasificadas en el rango de códigos C00-C97X correspondiente a neoplasias ${ }^{(38)}$. Se construyeron curvas de densidad para estudiar la estructura de edad entre las defunciones por cáncer y otras causas y, a partir de esa estructura, se estimaron tasas de mortalidad ajustada por edad por cada 100 mil habitantes a nivel municipal y por región, lo que permite una medida estándar de comparación. 
Adicionalmente, para detectar causas específicas de mortalidad por cáncer para cada región del estado, en esta segunda fase se estima la medida de concentración espacial relativa denominada, en este trabajo, índice local de defunción (ILD) para cada año en el periodo 1998-2012, lo que permite observar también la trayectoria histórica del indicador. Esta es una medida que retoma el principio de los indicadores de localización ${ }^{[b]}$ de la actividad económica, desarrollados en el contexto de la bibliografía en análisis regional(39). No obstante, dado su potencial para detectar patrones de comportamiento espacial, es una medida que se ha considerado en el campo de la bioestadística y en estudios empíricos de corte epidemiológico, tal como se documenta por los aportes de Beyene y Moineddin ${ }^{(40)}$, Moineddin et al. ${ }^{(41)}$ o Wright ${ }^{(42)}$.

En el presente contexto se aplica contemplando la referencia geográfica de los microdatos disponibles sobre cáncer, en México. El índice se define como:

$\operatorname{IDL}=\left(\frac{\mathrm{c}}{\mathrm{T}}\right)_{r} /\left(\frac{\mathrm{c}}{\mathrm{T}}\right)_{\mathrm{S}}$

Donde $c$ indica defunciones para una causa en particular dentro del rango C00-C97x; $T$ corresponde a la suma total de las defunciones en el rango C00-C97x. Los subíndices $r$ y $s$ aluden al ámbito geográfico regional y estatal respectivamente. Este indicador captura la incidencia en un espacio geográfico en términos proporcionales controlando el tamaño de su población respecto a la incidencia observada en un espacio geográfico base; en este caso se evalúa cada una de las cinco regiones respecto a la entidad. Dos umbrales del índice se consideran como referencia: IDL $>1$, indica una concentración superior del padecimiento estudiado respecto al nivel observado en el contexto estatal para la i-ésima región. De forma paralela $\mathrm{IDL}<1$ implica una ocurrencia de menor proporción en la región respecto al estado. Este indicador permitirá distinguir las causas específicas que se presentan con mayor incidencia en cada región del estado, lo que puede ser útil en el contexto de la presencia de actividades económicas específicas.

A partir de la información generada por el análisis exploratorio de datos se propone un modelo estadístico inferencial con la herramienta de regresión logística para evaluar la importancia relativa de cada región considerando las características demográficas adicionales como el sexo y la edad por grupos. La especificación del modelo propuesto es la siguiente:

$$
Y=\frac{1}{1+e^{-\left(\alpha+\beta_{1}+\beta_{2}+\beta_{3}+\beta_{4}\right)}}
$$

La variable binaria dependiente $Y$ toma el valor 1 en caso de que la defunción se presente por cáncer-causas en el rango C00-C97x de la CIE-10- y 0 en caso contrario.

Las variables dependientes del modelo son: $\beta_{1}$ grupo de edad: 1 (0-14 años), 2 (15-64 años), 3 (65-74 años), 4 (75 y más); $\beta_{2}$ sexo: 1 (hombre), 2 (mujer); $\beta_{3}$ región: 1 (Sur), 2 (río Yaqui), 3 (Empalme), 4 (río Sonora), 5 (Norte). La regionalización se consideró con base en los criterios hidrológicos y la localización de la actividad minera en el estado.

La variable dicotómica clúster $\left(\beta_{4}\right)$ toma el valor 1 , en caso de que el sitio de residencia del individuo se encuentre en los municipios que integran zonas de concentración relativa por descargas de agua residual acorde con el modelo geoestadístico propuesto; $y$, el valor 0 , en caso contrario.

\section{RESULTADOS}

\section{Calidad de los recursos hídricos}

Tal como se ha documentado, un nivel de análisis relevante por sus implicaciones sobre las condiciones de salud es el referente a las actividades que tienen impacto sobre la calidad de los recursos hídricos, en tanto constituyen un mecanismo de transmisión que posibilita la exposición de la población a elementos contaminantes. 


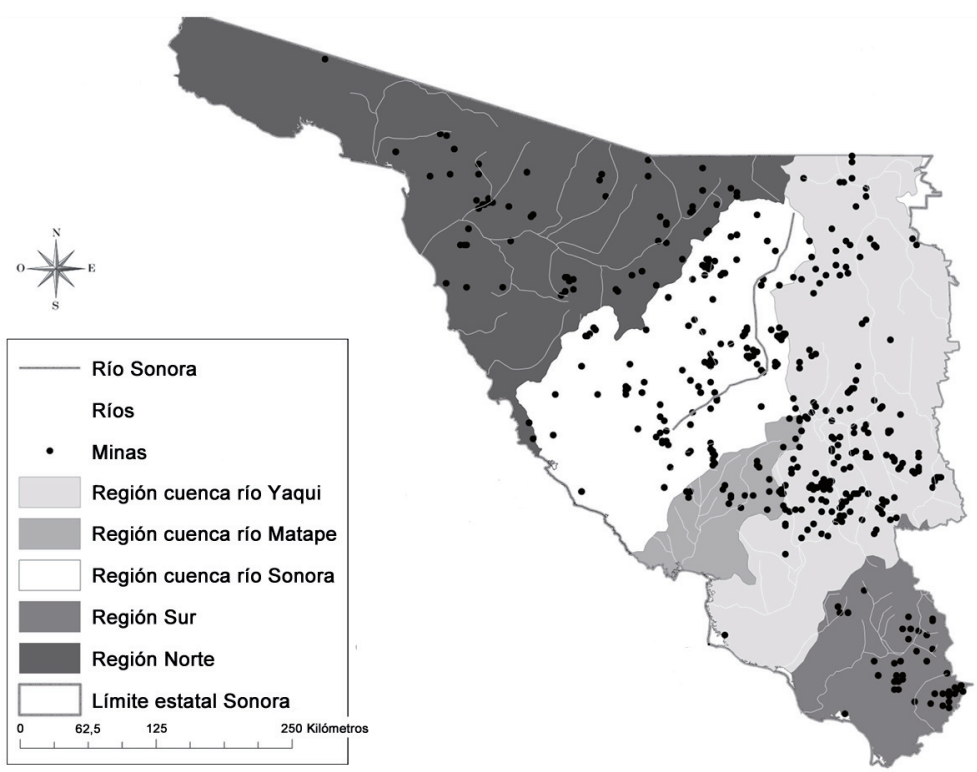

Figura 2. Regionalización para medir las externalidades negativas de la actividad económica. Estado de Sonora, México, 2015.

Fuente: Elaboración propia a partir de datos del Instituto Nacional de Estadística y Geografía (INEGI) y la Comisión Nacional para el Conocimiento y Uso de la Biodiversidad (CONABIO).

Para conducir el análisis de las externalidades de la actividad económica sobre los recursos hídricos se propone la clasificación de la entidad en cinco regiones tomando como base el vínculo entre proximidad de sitios de producción minera y la división hidrológica del estado por cuencas. Las regiones propuestas son:

- Región 1 (Sur): integrada por las cuencas de los ríos Mayo, Fuerte y el Estero de Bacorehuis, con 43 minas.

- Región 2 (río Yaqui): integrada por la cuenca del río Yaqui, con 173 minas.

- Región 3 (Empalme): integrada por la cuenca del río Matapé, con 27 minas.

- Región 4 (río Sonora): integrada por las cuencas del río Sonora y río Bacoachi, con 121 minas.

- Región 5 (Norte): integrada por las cuencas Bacanora-Mejorada, río Colorado, Desierto de Altar-Río Bamorí, Río Concepción-Arroyo Cocaspera, San Ignacio, con 64 minas.
La Figura 2 muestra la regionalización propuesta con base en los criterios hidrológicos y la localización de la actividad minera en el estado y, la Figura 3, la cuenca del río Sonora y su área de influencia en términos de la demarcación municipal. En total, en Sonora existen 428 minas, de las cuales 138 son minas de oro, 97 de plata, 58 de cobre y 8 de carbón.

La Figura 4 muestra la distribución espacial de las concesiones inscritas en el Registro Público de Derechos de Agua en la categoría de descargas, aprovechamientos que inciden en la calidad de los recursos hídricos a nivel local y de importancia por sus implicaciones desde la perspectiva de salud ambiental. Para mayor referencia, se incluyen dos radios de influencia con una circunferencia de $70 \mathrm{~km}$, tomando como criterio para su delimitación la densidad de concesiones alrededor de las dos principales ciudades del estado: Hermosillo y Obregón, en el sur. 


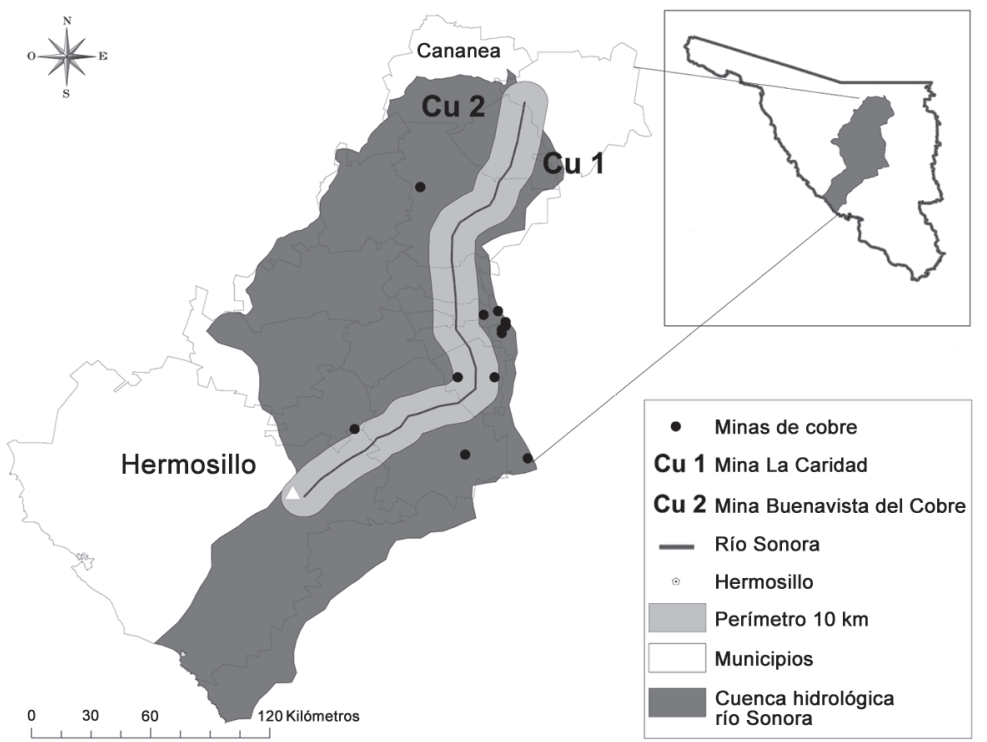

Figura 3. Regionalización para medir las externalidades negativas de la actividad económica. Cuenca del río Sonora, México, 2015.

Fuente: Elaboración propia a partir de datos del Instituto Nacional de Estadística y Geografía (INEGI) y la Comisión Nacional para el Conocimiento y Uso de la Biodiversidad (CONABIO).

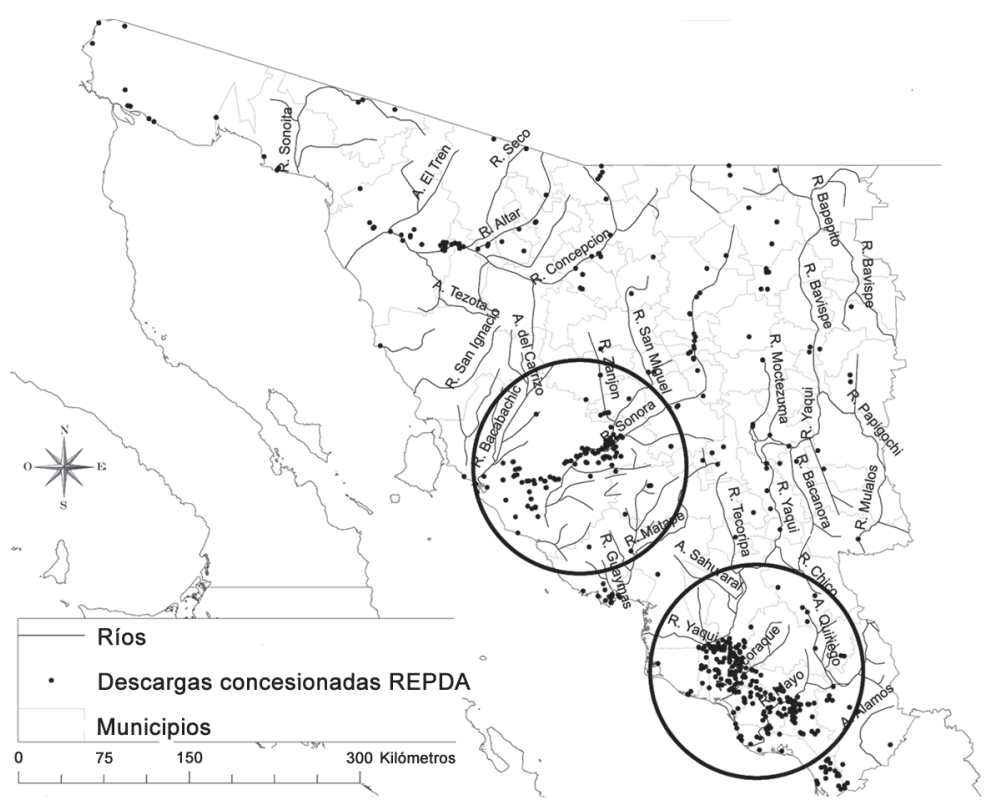

Figura 4. Distribución espacial de aprovechamientos por descargas de agua residual. Estado de Sonora, México, 2015.

Fuente: Elaboración propia a partir de datos de la Comisión Nacional del Agua (CONAGUA). 


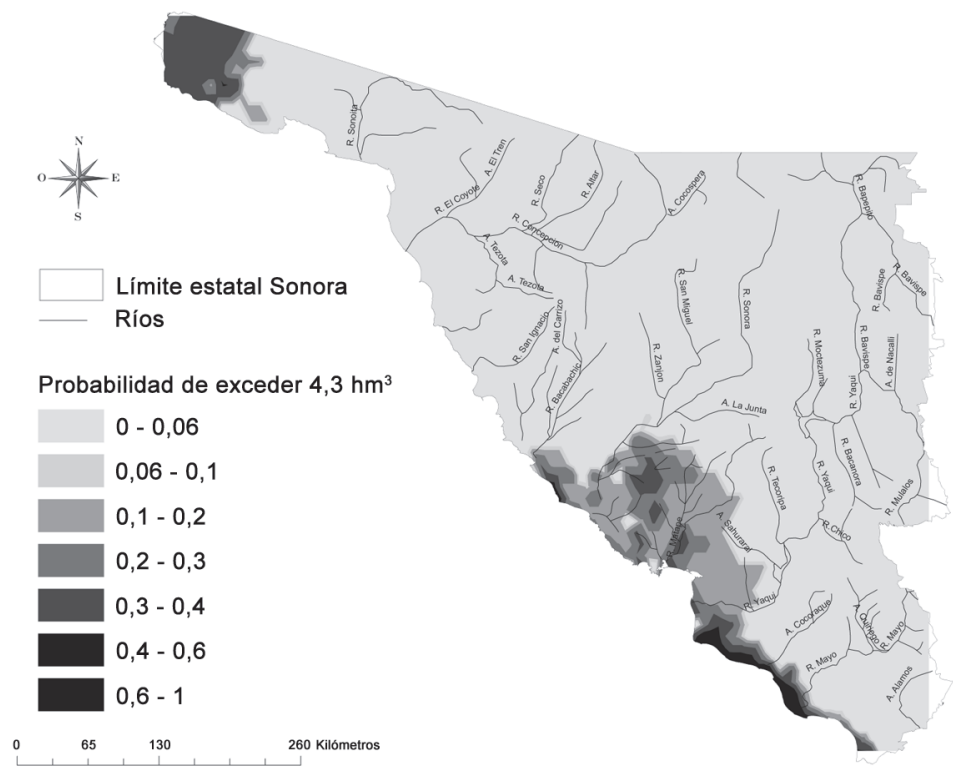

Figura 5. Distribución espacial de aprovechamientos por descargas de agua residual. Estado de Sonora, México, 2015.

Fuente: Elaboración propia a partir de datos de la Comisión Nacional del Agua (CONAGUA).

La Figura 5 muestra el resultado del modelo geoestadístico para la densidad de concesiones por descargas de agua residual, lo que permite detectar las áreas de concentración relativa en el estado.

La clasificación de las descargas inscritas por tipo permite distinguir que los principales emisores en el estado son el uso acuícola y el industrial, que representan el $54,7 \%$ y el $41,1 \%$ del volumen total de descargas concesionado respectivamente; mientras que los tipos público urbano, pecuario, servicios y agrícola representan, en conjunto, el 4,2\% restante. Esta asignación de las concesiones permite enfatizar la importancia de la actividad industrial en la que la minería, como se ha discutido, tiene un papel central $y$, al mismo tiempo, los resultados indican el impacto de una dinámica actividad acuícola que se desarrolla en los municipios a lo largo del golfo de California, entre los que Guaymas es el principal municipio por su volumen concesionado para realizar descargas de agua residual.
Además, el modelo geoestadístico generado confirma la existencia de un clúster de descargas residuales que incluye los municipios de Hermosillo, Guaymas y Huatabampo en la costa del pacífico. Esta distribución es de interés ya que permite distinguir que la localización de las concesiones por descargas ejerce presión sobre tres corrientes superficiales adyacentes a concentraciones urbanas: el río Sonora, el río Yaqui en las proximidades de Obregón, un importante centro industrial del estado, y el río Mayo en los linderos de la ciudad de Navojoa.

\section{Defunciones por cáncer en el estado de Sonora}

En esta sección se explora la distribución espacial de las defunciones por cáncer en el estado de Sonora. Como se ha identificado en la sección precedente, en el estado de Sonora, los efectos de las actividades económicas específicas como la minería ejercen 
presión sobre la calidad de los recursos hídricos en las inmediaciones de importantes centros urbanos de la entidad. Una implicación social de estos efectos se expresa a través de las condiciones de salud de la población. En este contexto, un vínculo de interés particular es el documentado por la incidencia de cáncer ante la exposición de largo plazo a elementos contaminantes encontrados en sitios de actividad minera ${ }^{(20,43)}$, una relación en la que prevalece un entendimiento rudimentario, si bien se reconoce que no implica causalidad directa debido a la existencia de múltiples factores de riesgo involucrados.

La Figura 6 muestra la evolución histórica de las defunciones por cáncer en el estado, en la última década mientras que la Figura 7 muestra la estructura de edad en la que ocurrieron las defunciones por cáncer en el estado de Sonora en 2014.

Las curvas de densidad para la distribución de las edades de las defunciones en Sonora revelan que el cáncer es un padecimiento que muestra una mayor incidencia en la etapa entre los 37 y los 78 años y alcanza su mayor nivel hacia los 74 en promedio. Vemos que es una causa de muerte que afecta la etapa productiva de los individuos de forma clara. Esta distinción se hace evidente también por la brecha existente después de los 78 años, en la que el resto de las causas de mortalidad (categoría otras causas) tiene mayor ocurrencia. Es interesante observar que, para la población en edad infantil, predomina la incidencia de otras causas.

Para controlar por la brecha en la distribución de edades que se ha encontrado, se estimaron las tasas de mortalidad ajustadas por la estructura de edad en cada una de las cinco regiones propuestas del estado, usando el método directo ${ }^{(44)}$ y la estructura de edades en la entidad como la población estándar (Figura 8). En cuanto a los resultados de la estimación del índice local de defunción, la tasa de mortalidad ajustada por edad para las categorías C00-C97X correspondientes a defunciones por neoplacias para el estado de Sonora es de 87 personas por cada 100 mil habitantes, cifra superior a la

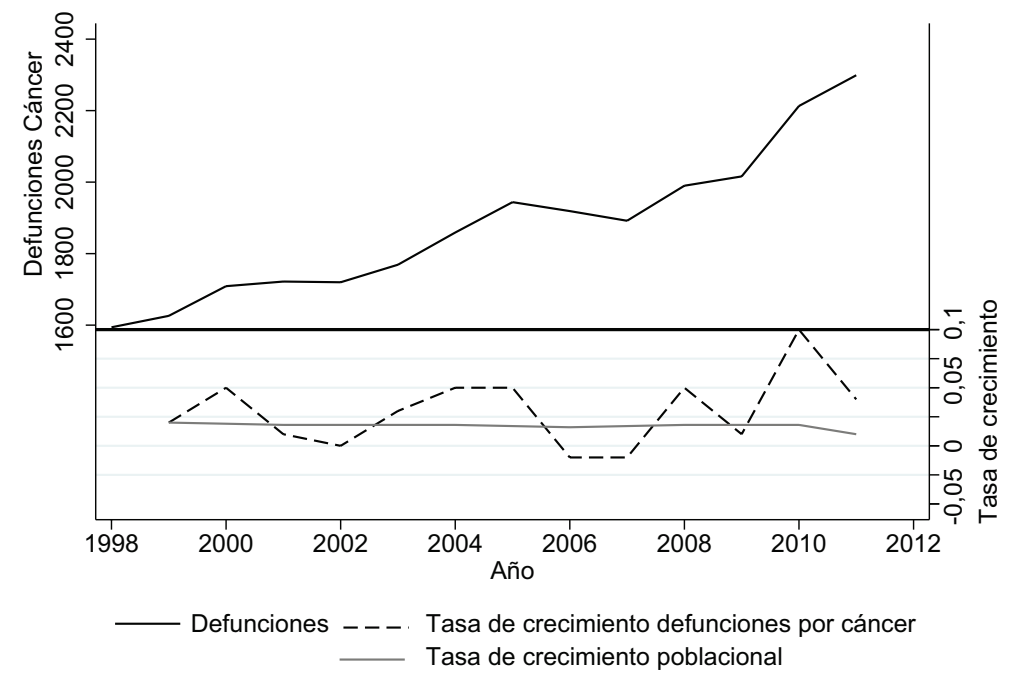

Figura 6. Defunciones por cáncer. Estado de Sonora, México, 19982011.

Fuente: Elaboración propia a partir de datos del Sistema Nacional de Información en Salud (SINAIS) y datos de población del Instituto Nacional de Estadística y Geografía (INEGI). 


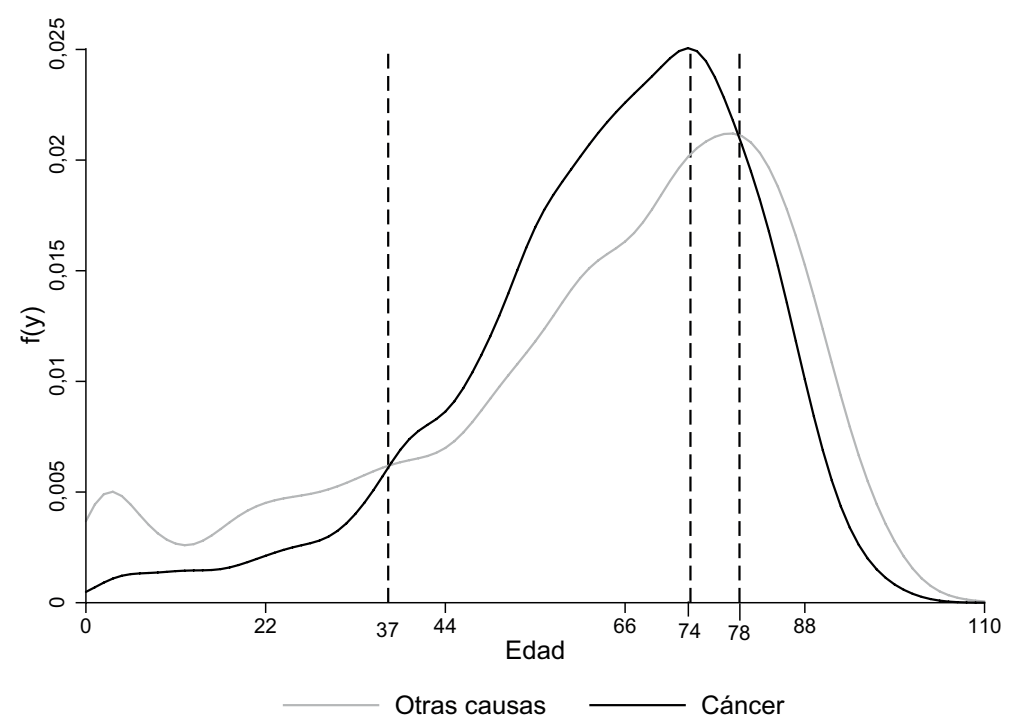

Figura 7. Distribución de las defunciones por cáncer y otras causas, según edad. Estado de Sonora, México, 2011.

Fuente: Elaboración propia a partir de datos del Sistema Nacional de Información en Salud (SINAIS) y datos de población del Instituto Nacional de Estadística y Geografía (INEGI).

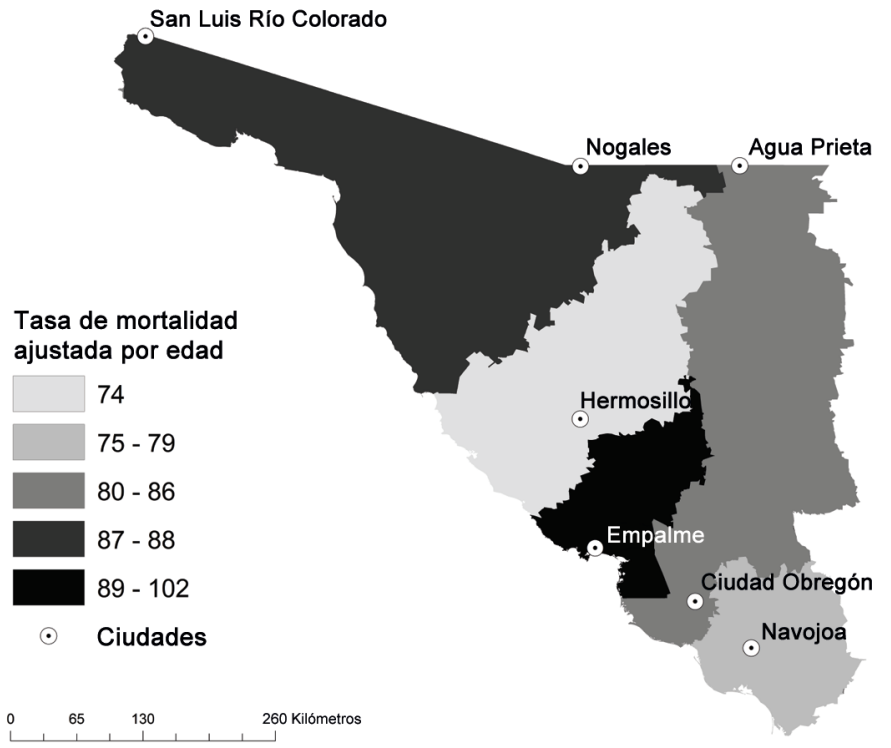

Figura 8. Tasas de mortalidad ajustada por edad según región. Estado de Sonora, México, 2011.

Fuente: Elaboración propia a partir de datos del Sistema Nacional de Información en Salud (SINAIS). 
estimación para el contexto nacional en el mismo periodo, con 69 personas por cada 100 mil habitantes según la Organización Mundial de la Salud ${ }^{(45)}$ e inferior al escenario en el vecino estado de Arizona que es de 156 personas por cada 100 mil habitantes en el periodo 2007-2011 $1^{(46)}$.

Por otro lado, la estimación permite distinguir una clara diferencia para el comportamiento a lo largo del estado, con la región 3 (Empalme), que presenta las tasas de mortalidad de mayor magnitud. Por su parte, la estimación del ILD permite desagregar para cada región las causas específicas que

Tabla 1. Modelo de regresión logística para las defunciones por cáncer. Estado de Sonora, México, 2014.

\begin{tabular}{|c|c|c|c|}
\hline Variables & OR & Valor de $p$ & IC95\% \\
\hline \multicolumn{4}{|l|}{ Sexo } \\
\hline Hombre $^{1}$ & - & - & - \\
\hline Mujer & 1,20 & 0,00 & 1,$14 ; 1,37$ \\
\hline \multicolumn{4}{|l|}{ Grupo de edad } \\
\hline $0-14^{1}$ & - & - & - \\
\hline $15-64$ & 3,83 & 0,00 & 2,$79 ; 5,25$ \\
\hline $65-74$ & 4,51 & 0,00 & 3,$27 ; 6,22$ \\
\hline+75 & 2,67 & 0,00 & $1,94,3,67$ \\
\hline \multicolumn{4}{|l|}{ Región } \\
\hline Región Sur ${ }^{1}$ & - & - & - \\
\hline Región río Yaqui & 1,12 & 0,14 & 0,$96 ; 1,31$ \\
\hline Región Empalme & 1,08 & 0,48 & 0,$88 ; 1,32$ \\
\hline Región río Sonora & 1,27 & 0,00 & 1,$09 ; 1,48$ \\
\hline Región Norte & 1,15 & 0,11 & 0,$97 ; 1,36$ \\
\hline \multicolumn{4}{|l|}{ Cluster } \\
\hline Fuera $^{1}$ & - & - & - \\
\hline Dentro & 1,17 & 0,02 & 1,$02 ; 1,33$ \\
\hline
\end{tabular}

Fuente: Elaboración propia a partir del Sistema Nacional de Información en Salud.

${ }^{1}$ Valor de referencia.

OR= Odds ratio; IC95\%= intervalo de confianza del $95 \%$.

Nota: La selección de la especificación del modelo se realizó con base en las pruebas de mejor ajuste de Hosmer y Lemershow, así como también se realizaron las pruebas estándar para descartar problemas de multicolinealidad y errores de especificación en la función de enlace entre variable dependiente y los regresores. ocurren con una proporción relativa superior al nivel observado en el estado. Se identificaron las siguientes causas por región:

- Región 1 (Sur): C32 (tumor maligno de la laringe); C76 (tumor maligno de otros sitios y de sitios mal definidos); y C90 (mieloma múltiple y tumores malignos de células plasmáticas).

- Región 2 (río Yaqui): D38 (tumor de comportamiento incierto o desconocido del oído medio y de los órganos respiratorios e intratorácicos); C53 (tumor maligno del cuello del útero); C61 (tumor maligno de la próstata).

- Región 3 (Empalme): C45 (mesotelioma); C71 (tumor maligno del encéfalo); C23 (tumor maligno de la vesícula biliar).

- Región 4 (río Sonora): C34 (tumor maligno de los bronquios y del pulmón); C16 (tumor maligno del estómago); C18 (tumor maligno del colon).

- Región 5 (Norte): C25 (tumor maligno del páncreas); C64 (tumor maligno del riñón); C15 (tumor maligno del esófago).

Si bien la fase exploratoria de datos ha permitido evidenciar rasgos importantes para estudiar esta relación, se propone la aplicación de métodos inferenciales con el objeto de examinar las diferencias entre cada región del estado y su interacción con las características de la población como la edad y el sexo.

La Tabla 1 muestra los resultados del análisis inferencial que considera como variable dependiente las defunciones por cáncer en el estado.

Para la variable sexo se encuentra que la probabilidad de un evento por cáncer registra un incremento estadísticamente significativo para el sexo femenino respecto al sexo masculino (OR $=1,2 ;$ IC95\% $[1,14 ; 1,37])$. Considerando la variable grupo de edad se observa que el grupo con mayor probabilidad de un evento por cáncer se encuentra en la categoría 3 (65-74 años de edad) $(\mathrm{OR}=4,51$; IC95\% [3,27; 6,22]), lo que confirma el análisis exploratorio realizado en la sección precedente. Se halla también que el incremento observado para la categoría 4 (75 años y más) 


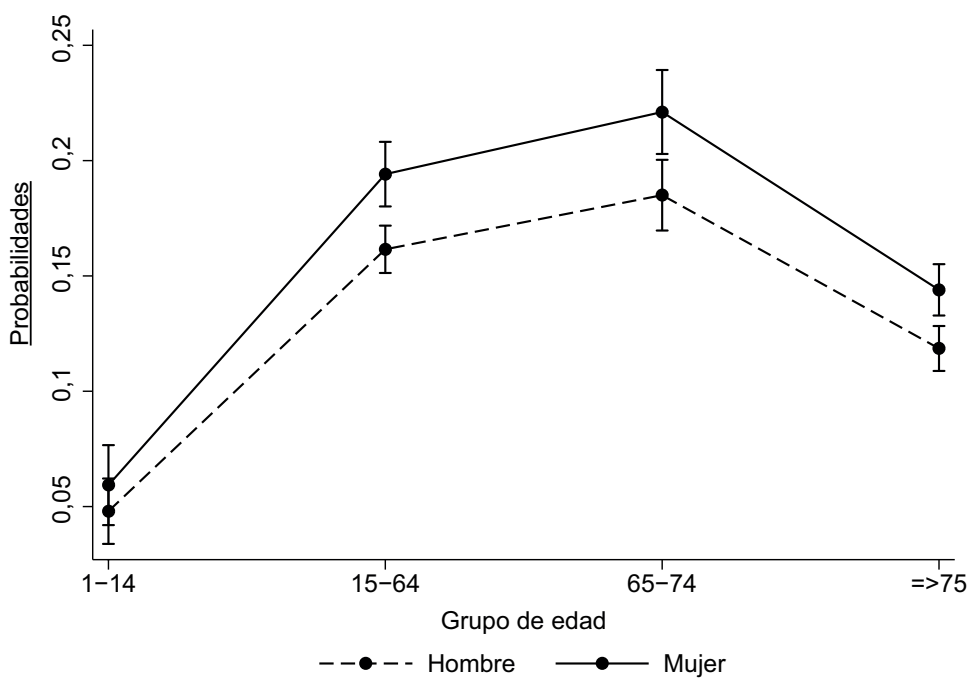

Figura 9. Probabilidades estimadas para defunciones por cáncer, según sexo. Estado de Sonora, México, 2014.

Fuente: Elaboración propia a partir de datos del Sistema Nacional de Información en Salud (SINAIS).

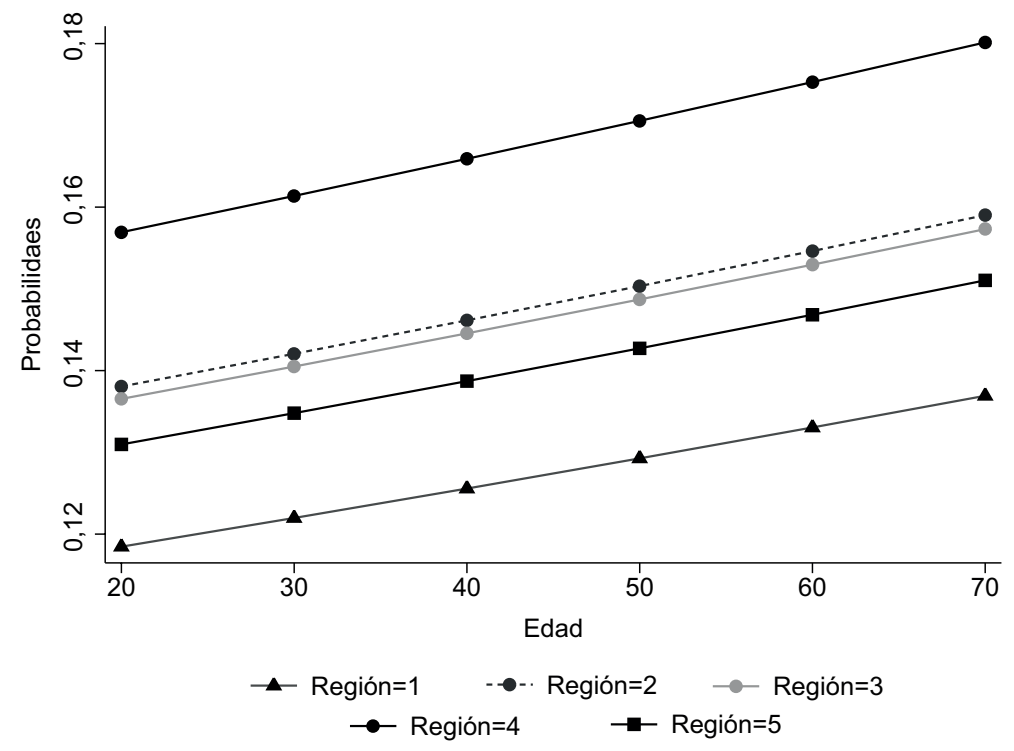

Figura 10. Probabilidades estimadas para defunciones por cáncer, según región. Estado de Sonora, México, 2014.

Fuente: Elaboración propia a partir de datos del Sistema Nacional de Información en Salud (SINAIS). 
es inferior a la categoría precedente, lo que implica que en este grupo de edad son otras las causas que prevalecen en contraste con el grupo de 65 a 74 años.

Para la variable clúster que agrupa a los municipios detectados por la actividad de descargas de agua residual se halla un odds ratio de 1,17 (IC95\% [1,02; 1,33]), por lo que la residencia en los municipios que integran el clúster se asocia con un incremento en la posibilidad de fallecimiento por cáncer.

La variable región permite distinguir dos hallazgos relevantes: el primero es que, aparentemente, no hay una diferencia estadísticamente significativa entre el odds ratio para la regiones 1, 2, 3 y 5; el segundo, indica que la probabilidad de un evento por cáncer entre la región 1 (Sur), la categoría de referencia y la región 4 (río Sonora) se incrementa notoriamente $(\mathrm{OR}=1,27$; IC95\% $[1,09 ; 1,48])$. Para tener una perspectiva gráfica de estos hallazgos se estima la evolución de las probabilidades mediante los efectos marginales con la interacción entre las variables grupo de edad, sexo y región (Figura 9 y Figura 10).

El cálculo muestra la mayor probabilidad que se registra para el sexo femenino y su evolución a medida que se incrementa la edad alcanzando su nivel máximo en el rango de edad entre 64 y 74 años. Por otro lado, las probabilidades difieren para cada región en un rango que va del $12 \%$ al $18 \%$ y alcanzan su máximo nivel en la región 4 (río Sonora). Estimaciones en el contexto internacional del National Cancer Institute ${ }^{(47)}$ indican una probabilidad de fallecimiento por cáncer en el periodo 2009-2011 del 17,7\% para la población hispana en EE.UU.

\section{DISCUSIÓN DE RESULTADOS Y CONCLUSIONES}

En la relación entre actividad económica y medio ambiente se examinó la distribución espacial de la actividad minera y las concesiones por descargas de agua residual, actividades que tienen impacto sobre la calidad de los recursos hídricos y constituyen un mecanismo de transmisión que posibilita la exposición de la población a elementos contaminantes.

La discusión se desarrolló bajo el paradigma teórico que enfatiza la incidencia de condiciones de salud como el resultado de una producción social ${ }^{(48)}$ en la que la actividad económica es un detonante central por la generación de externalidades negativas susceptibles de afectar las condiciones de salud de la población.

El presente trabajo concuerda con antecedentes realizados por autores como Bundschuh et al. ${ }^{(23)}$ quienes desde el contexto de la salud pública para Latinoamérica aseveran que actividades antropogénicas como la minería, al facilitar la movilización de contaminantes de ocurrencia natural, representan un problema con implicaciones de salud pública ${ }^{(23)}$. Por otro lado, a pesar de que autores como Ebenstein ${ }^{(49)}$ han documentado previamente la relación entre el incremento de cáncer en órganos digestivos y el deterioro de la calidad del agua, su propuesta difiere del presente enfoque, entre otros elementos, porque considera el proceso de desarrollo económico como un factor detonador y retoma para su validación empírica el caso de países con acelerado ritmo de crecimiento de la actividad económica en la última década, como lo es China. Uno de los retos de ese enfoque es el control de posibles efectos por exposición a comportamientos de riesgo y tradicionalmente determinantes sociales como la dieta, el uso de tabaco y la exposición a contaminantes en el aire, que influyen en la incidencia de padecimientos como el cáncer. Así mismo, los resultados del presente trabajo, abren la posibilidad para complementarse con esfuerzos adicionales de investigación que aporten evidencia sobre la relación de causalidad sugerida.

Tal como se documenta en el presente trabajo las variaciones regionales en los niveles observados de los indicadores de salud permiten aproximar diferentes grados de vulnerabilidad de la población y esta información representa un insumo para el diseño de estrategias preventivas, que no se limita a actividades manufactureras o extractivas 
tradicionales como la minería. No obstante, su estudio en el ámbito de la minería adquiere pertinacia ante la necesidad de comprender mejor los impactos de contingencias a nivel local en el largo plazo.

Los elementos complementarios que presenta Colborn ${ }^{(50)}$, desde una perspectiva de salud pública, muestran el potencial del enfoque multidisciplinario sugerido por este trabajo. El autor indaga actividades económicas emergentes como la extracción de gas natural no convencional (shale gas) mediante la técnica denominada fracturación hidráulica, técnica intensiva en el uso de recursos hídricos y que, de acuerdo con Myers ${ }^{(51)}$, presenta riesgos potenciales de contaminación de las fuentes de abastecimiento de agua para consumo humano.

Esta es un área de interés que muestra la utilidad de la aplicación del paradigma propuesto en este trabajo para diversos países de América Latina, como Venezuela, Brasil, México o Argentina, país que alberga el nivel potencial de reservas de gas natural no convencional más importante del continente americano, en las inmediaciones de la cuenca de Neuquén, principalmente ${ }^{(52)}$. De modo que abordar las implicaciones locales puede ser de interés bajo una concepción amplia de los factores que determinan las condiciones de salud de la población, y el marco analítico aplicado en el presente trabajo ofrece una alternativa para su aproximación.

Si bien ciertas propuestas, como el análisis de Mactaggart ${ }^{(53)}$, enfatizan incluso la distinción del grado de vulnerabilidad de la población en función del carácter rural o urbano del espacio de análisis, en el presente trabajo este aspecto no se aborda de forma explícita más allá de la delimitación regional con base en criterios hidrológicos, por lo que este aspecto se percibe como una oportunidad de investigación para futuros trabajos.

En el presente estudio, la regionalización propuesta basada en la distribución por cuencas hidrográficas permitió establecer diferencias en las tasas de mortalidad ajustadas por edad por cáncer con la región 3 (Empalme) que comprende los municipios de Empalme, Guaymas, La Colorada, Mazatán y
Villa Pesqueira, con las tasas de mayor magnitud en el estado, con 102 defunciones por cáncer por cada 100 mil habitantes, nivel superior a la estimación para el contexto nacional en el mismo periodo que es de 69 personas por cada 100 mil habitantes, según la $\mathrm{OMS}^{(45)}$, e inferior al escenario en el vecino estado de Arizona que es de 156 personas por cada 100 mil habitantes, en el periodo 2007-2011 ${ }^{(46)}$.

Al mismo tiempo, el análisis de concentración espacial propuesto permitió observar el comportamiento al interior de la región de las causas de mortalidad por cáncer identificadas. Al respecto, en la región 3 (Empalme), se encuentra una particular alta incidencia de casos en la clasificación C45 correspondiente a mesotelioma, un tipo de cáncer cuyo vínculo con actividades productivas tiene un consenso documentado ${ }^{(54)}$; lo que es un indicativo de áreas de posible intervención en el plano de diseño de estrategias de monitoreo en la relación economía-salud en la entidad.

La modelación con herramientas de corte inferencial permite determinar que los ocho municipios que integran el clúster de descargas de aguas residuales muestran una mayor probabilidad de presentar defunciones por cáncer en relación con los sitios en el estado que están fuera de esta zona. Así mismo, se identificó que las probabilidades asociadas a la muerte por cáncer en el estado de Sonora difieren para cada región en un rango que va del $12 \%$ al $18 \%$ y alcanzan su máximo nivel en la región 4 (río Sonora), resultado que es comparable con estimaciones sobre la población hispana en EE.UU. que documentan una probabilidad de muerte por cáncer en el periodo 2009-2011, del 17,7\% ${ }^{(39)}$.

Para la región 4 (río Sonora) se halló una concentración relativa de la ocurrencia de defunciones en cáncer por: tumor maligno de los bronquios y del pulmón, tumor maligno del estómago y tumor maligno del colon. $\mathrm{Si}$ bien en el primer caso se ha mostrado una relación con la exposición a elementos contaminantes como arsénico ${ }^{(20)}$, establecer una relación de causalidad con niveles de exposición particulares requiere de la realización 
de investigaciones subsecuentes dada la multiplicidad de factores de riesgo asociados.

No obstante, ante la presencia de elementos químicos como el arsénico en el área de influencia de la contingencia minera ocurrida en Cananea, en 2014, ubicada dentro de la región 4 (río Sonora) delineada en el presente trabajo, con un nivel de 42,7 $\mathrm{mg} / \mathrm{I}^{(27)}$, que rebasa el límite permisible en agua para consumo humano que es de 0,05 mg/l, según la Norma Oficial Mexicana NOM-127-SSA1-1994, se concluye que es de interés para la región la propuesta de ajuste de los parámetros de dicha norma, como se proyecta en la NOM-250-SSA1-2014, que consigna un subconjunto de contaminantes comunes en sitios con actividad minera.

Adicionalmente, el vínculo analizado entre fuentes de abastecimiento de recursos hídricos para consumo humano y la intensa actividad minera en el estado permiten sugerir la necesidad de la operación de una agencia regulatoria especializada que supervise el tema ambiental en la actividad minera, una figura que se tiene para otros sectores con alto potencial de generar externalidades negativas al medio ambiente como la extracción de hidrocarburos mediante la Agencia de Seguridad, Energía y Ambiente (ASEA) recientemente creada, y que provee un marco de análisis para atender la ocurrencia de eventualidades en un sector que, ciertamente, representa riesgos de emisión de residuos potencialmente nocivos para la salud.

En el contexto de la minería, esta figura propuesta podría generar condiciones favorables para comprender mejor los impactos de esta actividad económica y su interrelación con los mecanismos de transmisión en la presencia de fuentes de abastecimiento de agua potable.

\section{REFERENCIAS BIBLIOGRÁFICAS}

1. García y Alva F. México y sus progresos: Album-directorio del estado de Sonora. Hermosillo: Imprenta Oficial; 1905-1907.

2. Meyer E, (coord.). La lucha obrera en Cananea, 1906. 2d ed. México DF: Instituto Nacional de Antropología e Historia; 1990.

3. Sariego JL. Enclaves y minerales en el norte de México: historia social de los mineros de Cananea y Nueva Rosita, 1900-1970. México DF: CIESAS; 1988.

4. Sonnichsen CL. Colonel Greene and the copper skyrocket. Tucson: The University of Arizona Press; 1976.
5. Aguirre MJ. Cananea: Las garras del imperialismo en las entrañas de México. México: Editora B. Costa-Amic; 1958.

6. Bailey LR. Bisbee: Queen of the Copper Camps. Tucson: Westernlore Press; 1983.

7. Sprouse TW. Water issues on the ArizonaMexico border: The Santa Cruz, San Pedro and Colorado Rivers [Internet]. Tucson: Water Resources Research Center, University of Arizona; 2005 [citado 10 sep 2015]. Disponible en: https:// goo.gl/LAkF5K

8. Martinelli PC. Undermining race: ethnic identities in Arizona Copper Camps, 1880-1920. Tucson: University of Arizona Press; 2009. 
9. Schwantes CA. Bisbee: Urban out post on the frontier. Tucson: University of Arizona Press; 1992.

10. Spude RL. Mineral frontier in transition: Copper mining in Arizona, 1880-1885. New Mexico Historical Review. 1976;51:19-34.

11. Gracida JJ. El Sonora moderno (1892-1910). En: Calderón S, (coord). Historia general de Sonora. Vol. 4. Hermosillo: Gobierno del Estado de Sonora; 1985.

12. World Health Organization. The economics of the social determinants of health and health inequalities: a resource book. Geneva: WHO; 2013.

13. Pandey $P$, Sharma R, Roy M, Pandey M. Toxic mine drainage from Asia's biggest copper mine at Malanjkhand, India. Environmental Geochemistry and Health. 2007;29(3):237-248.

14. El Gharmali A, Rada A, El Adnani M, Tahlil N, El Meray M, Nejmeddine A. Impact of acid mining drainage on the quality of superficial waters and sediments in the Marrakesh region, Morocco. Environmental Technology. 2004;25(12):1431-1442.

15. Ramirez-Andreotta MD, Brusseau ML, Beamer $P$, Maier RM. Home gardening near a mining site in an arsenic-endemic region of Arizona: Assessing arsenic exposure dose and risk via ingestion of home garden vegetables, soils, and water. Science of the Total Environment. 2013;454-455:373-382.

16. Tsuji JS, Perez V, Garry MR, Alexander DD. Association of low-level arsenic exposure in drinking water with cardiovascular disease: A systematic review and risk assessment. Toxicology. 2014;323:78-94.

17. US Environmental Protection Agency. Arsenic in drinking water funding sources [Internet]. 2015 [citado 9 sep 2015]. Disponible en: http://goo.gl/ nMw10T.

18. Centers for Disease Control and Prevention. Arsenic [Internet]. 2009 [citado 14 sep 2015]. Disponible en: http://goo.gl/GgwHe4.

19. Agency for Toxic Substances and Disease Registry. Public Health Statement: Arsenic [Internet]. Atlanta: ATSDR; 2007 [citado 10 sep 2015]. Disponible en: http://goo.gl/8n74rr.

20. Organización Mundial de la Salud, International Agency for Research on Cancer. Arsenic and arsenic compounds. IARC Monographs on the Evaluation of Carcinogenic Risks to Humans. 1987; (suppl 7):100-106.
21. Hughes MF. Biomarkers of exposure: A case study with inorganic arsenic. Environmental Health Perspectives. 2006;114(11):1790-1796.

22. Razo I, Carrizales L, Castro J, Díaz-Barriga F, Monroy M. Arsenic and heavy metal pollution of soil, water and sediments in a semi-arid climate mining area in Mexico. Water, Air, and Soil PoIlution. 2004;152(1):129-152.

23. Bundschuh J, Litter MI, Parvez F, Román-Ross G, Nicolli HB, Jean JS, Liu CW, López D, Armienta MA, Guilherme LR, Cuevas AG, Cornejo L, Cumbal L, Toujaguez R. One century of arsenic exposure in Latin America: A review of history and occurrence from 14 countries. Science of The Total Environment. 2012;429(1):2-35.

24. Wilson B, Pyatt FB. Heavy metal dispersion, persistance, and bioccumulation around an ancient copper mine situated in Anglesey, UK. Ecotoxicology and Environmental Safety. 2007;66(2):224-231.

25. Gobierno de la República. Balance de las acciones del Gobierno de la República en el río Sonora [Internet]. 2015 [citado 21 octubre 2015]. Disponible en: http://goo.gl/0zeJ8k.

26. Procuraduría Federal de Protección al Ambiente. Impone PROFEPA multas por 22.9 MPD a empresa minera Buenavista del Cobre [Internet]. Hermosillo, Sonora: PROFEPA; 2015 [citado 10 oct 2015]. Disponible en: http://goo.gl/emsWEX.

27. Secretaría de Medio Ambiente y Recursos Naturales, Universidad Nacional Autónoma de México. Informe de avances de evaluación de la calidad del agua de la presa el Molinito [Internet]. 2015 [citado 8 oct 2015]. Disponible en: http:// goo.gl/ZcFp7L.

28. Estados Unidos Mexicanos, Secretaría de Salud. Norma Oficial Mexicana NOM-127-SSA1-1994, Salud Ambiental, Agua para uso y consumo humano: Límites permisibles de calidad y tratamientos a que debe someterse el agua para su potabilización. Diario Oficial de la Federación. 22 nov 2000.

29.World Health Organization. Preventing disease through healthy environments: Towards an estimate of the environmental burden of disease. Geneva: WHO; 2006.

30. Krieger N. Epidemiology and the web of causation: Has anyone seen the spider? Social Science \& Medicine. 1994;39:887-903.

31. Honjo K. Social epidemiology: Definition, history, and research examples. Environmental Health Preventive Medicine. 2004;9(5):193-199. 
32. Mallath MK, Taylor DG, Badwe RA, Rath GK, Shanta V, Pramesh Sullivan R. The growing burden of cancer in India: epidemiology and social context. The Lancet Oncology. 2014;15(6):e205-e212.

33. Dold B. Evolution of acid mine drainage formation in Sulphidic Mine Tailings. Minerals. 2014;4(3):621-641.

34. Isaaks E, Srivastava RM. An Introduction to applied geostatistics. North Carolina: Oxford University Press; 1989.

35. Pardo-Igúzquiza E, Chica-Olmo M, GarciaSoldado MJ, Luque-Espinar JA. Using semivariogram parameter uncertainty in hydrogeological applications. Ground Water. 2009;47:25-34.

36. Waller LA, Gotway CA. Spatial exposure data applied spatial statistics for public health data. New Jersey: John Wiley \& Sons; 2004.

37. Comisión Nacional del Agua. Registro Público de Derechos de Agua [Internet]. Ciudad de México: CONAGUA; 2015 [citado 21 sep 2015]. Disponible en: http://goo.gl/KKtJW2.

38. Dirección General de Información en Salud. Base de datos sobre defunciones [Internet]. Ciudad de México [citado 1 sep 2015]. Disponible en: http://goo.gl//1Bmg8.

39. Isard W. Métodos de análisis regional: Una introducción a la ciencia regional. Barcelona: Ediciones Aries; 1971.

40. Beyene J, Moineddin R. Methods for confidence interval estimation of a ratio parameter with application to location quotients. BMC Medical Research Methodology. 2005;5:32.

41. Moineddin R, Beyene J, Boyle E. On the location quotient confidence interval. Geographical Analysis. 2003;35:249-256.

42. Wright SE. The spatial distribution and geographic analysis of endodontic office locations at the national scale. Journal of Endodontics. 1994;20:500-505.

43. Alarcón-Herrera MT, Bundschuh J, Nath B, Nicolli HB, Gutierrez M, Reyes-Gomez VM, Nuñez D, Martín-Dominguez IR, Sracek O. Cooccurrence of arsenic and fluoride in groundwater of semi-arid regions in Latin America: Genesis, mobility and remediation. Journal of Hazardous Materials. 2013;262:960-969.

44. Naing N. Easy way to learn standardization: Direct and indirect methods. Malaysian Journal of Medical Sciences. 2000;7(1):10-15.
45. International Agency for Research on Cancer, World Health Organization. Cancer Mortality Database [Internet]. 2015 [citado 10 jun 2015]. Disponible en: http://goo.gl/IIWZ1s.

46. American Cancer Society. Cancer Facts \& Figures 2015 [Internet]. Atlanta: American Cancer Society; 2015 [citado 10 sep 2015]. Disponible en: http://goo.gl/4zHKUv.

47. National Center Institute. SEER Cancer Statistics Review 1975-2012 [Internet]. 2014 [citado 10 ago 2015]. Disponible en: http://goo.gl/CxV85D.

48. Krieger N. A glossary for social epidemiology. Journal of Epidemiology and Community Health. 2001;55(10):693-700.

49. Ebenstein A. Water Pollution and Digestive Cancers in China, Review of Economics and Statistics. 2012;94(1):186-201.

50. Colborn T, Kwiatkowski C, Schultzz K, Bachran M. Natural gas operations from a public health perspective. Human and Ecological Risk Assessment: An International Journal. 2011;17(5):1039-1056.

51 Myers T. Potential contaminant pathways from hydraulically fractured shale to aquifers. Ground Water. 2012;50(6):872-882.

52. Energy Information Administration, Advanced Resources International. Technically recoverable shale oil and shale gas resources: an assessment of 137 shale formations in 41 countries outside the United States [Internet]. 2013 [citado $10 \mathrm{sep}$ 2015]. Disponible en: http://goo.gl/ZqZAI9.

53. Mactaggart F, McDermott L, Tynan A, Gericke C. Examining health and well-being outcomes associated with mining activity in rural communities of high-income countries: A systematic review. Australian Journal of Rural Health. 2016. doi: 10.1111/ajr.12285.

54. Fernández Francés J. Cáncer de pleura. Mesotelioma. Medicine. 2014;11(67):3995-4000.

\section{NOTAS FINALES}

[a] En el ámbito social, el concepto alude a la existencia de impactos (positivos o negativos) sobre terceros, comúnmente asociados con la realización de actividades económicas. Su origen se puede rastrear en el trabajo pionero del profesor Ronald Coase, Premio Nobel de Economía 1991. En el presente trabajo se emplea para hacer referencia a los impactos adversos sobre condiciones de salud de la población, que representan costos 
sociales por lo que se asume que constituyen una expresión de externalidades negativas. El concepto no se limita a su connotación de externalidades negativas. En el contexto de la salud pública, una externalidad positiva clásica es el beneficio para la comunidad derivado de las campañas de vacunación, ya que la aplicación de una vacuna y su efecto al evitar la transmisión de un padecimiento hacia la comunidad representa un beneficio indirecto o externalidad positiva. [b] En el contexto de la literatura de análisis regional, una medida de contraste para medir la concentración relativa de actividades económicas es el denominado location quotient (LQ). Esta técnica ha sido aplicada con interés en el campo de la bioestadística recientemente y se retoma en el presente trabajo a partir de la referencia geográfica de los microdatos estudiados.

\section{FORMA DE CITAR}

Manzanares Rivera JL. Calidad de los recursos hídricos en el contexto de la actividad económica y patrones de salud en Sonora, México. Salud Colectiva. 2016;12(3):397-414. doi: 10.18294/sc.2016.811

Recibido: 4 de diciembre de 2015 | Versión final: 26 de abril de 2016 | Aprobado: 4 de mayo de 2016

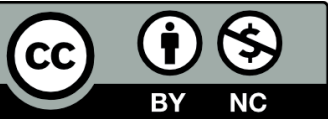

http://dx.doi.org/10.18294/sc.2016.811

Este obra está bajo una licencia de Creative Commons Reconocimiento-NoComercial 4.0 Internacional. Reconocimiento - Permite copiar, distribuir y comunicar públicamente la obra. A cambio, se debe reconocer y citar al autor original. No Comercial - Esta obra no puede ser utilizada con finalidades comerciales, a menos que se obtenga el permiso. 